\title{
Modelado numérico de pérdidas en conductores de sección rectangular mediante análisis por elementos finitos
}

\author{
I. Lope1, C. Carretero1, J. Acero1, R. Alonso2, J.M. Burdio1 \\ ${ }_{1}$ Grupo de Electrónica de Potencia y Microelectrónica (GEPM) \\ Instituto de Investigación en Ingeniería de Aragón (I3A) \\ Universidad de Zaragoza, Mariano Esquillor s/n, 50018, Zaragoza, Spain. \\ Tel.+34-976762707, Fax+34-976762043, e-mail: nlope@unizar \\ ${ }_{2}$ Grupo de Tecnologías Fotónicas (GTF) \\ Instituto de Investigación en Ingeniería de Aragón (I3A)
}

Universidad de Zaragoza, Mariano Esquillor s/n, 50018, Zaragoza, Spain.

\begin{abstract}
En esta ponencia, se presenta un método para calcular las pérdidas en alterna (incluyendo efecto skin y proximidad) en dispositivos magnéticos planares con sección de conducción rectangular. Se desarrolla un modelado de pérdidas similar al empleado con conductores de sección circular para los conductores anteriormente mencionados. El objetivo es obtener un proceso de diseño adecuado, especialmente para los dispositivos magnéticos implementados en placa de circuito impreso ( $\mathrm{PCB}$ ). Básicamente, el modelado de pérdidas está basado en la superposición de las pérdidas en el conductor (pérdidas de conducción y de proximidad), y también en una descomposición ortogonal del campo bajo el que se encuentran los conductores. El número de funciones necesarias para calcular las pérdidas de proximidad se reduce a una, tal y como ocurre en los conductores circulares. Esta función, junto con la función que describe las pérdidas de conducción, ambas contienen las dependencias geométricas y frecuenciales, son calculadas y tabuladas mediante un Análisis por Elementos Finitos (FEA). El campo magnético se calcula mediante una simulación magnetostática basada en FEA. De esta forma las pérdidas son obtenidas con una reducción del tiempo de cálculo considerable. Finalmente, se han caracterizado varios prototipos para verificar el modelado de pérdidas.
\end{abstract}

